Artigo Original

Original Article

Evelyn Vanessa Silva Souza' 1 lara Barreto Bassi² (C) Ana Cristina Côrtes Gama ${ }^{3}$ (B)

Descritores Voz Docentes Disfonia Dosimetria Fonoaudiologia

Keywords

Voice Faculty Dysphonia Dosimetry Speech, Language and Hearing Sciences

Endereço para correspondência: Evelyn Vanessa Silva Souza Av. Alfredo Balena, 190, sala 249, Santa Efigênia, Belo Horizonte (MG), Brasil, CEP: 30130-100.

E-mail: evelyn.souza.fono@gmail.com

Recebido em: Abril 09, 2020

Aceito em: Setembro 06, 2020

\section{Amplificador de voz: efeitos na dose e na intensidade vocal de professoras sem disfonia}

\author{
Voice amplifier: effects on dose and vocal \\ intensity of teachers without dysphonia
}

\section{RESUMO}

Objetivo: Analisar a interferência do uso do amplificador de voz na dose vocal de professoras não disfônicas Método: Trata-se de um estudo experimental, comparativo intrassujeitos, composto por 20 professoras do ensino fundamental da Rede Municipal de Ensino de Belo Horizonte/MG. Após o consentimento as participantes, foram solicitadas a responder o questionário de Escala de Sintomas Vocais - ESV e posteriormente participaram de dois momentos do estudo, selecionados aleatoriamente. No primeiro momento as participantes utilizaram somente o dosímetro vocal e no segundo momento utilizaram o dosímetro vocal e o amplificador de voz. As medições foram registradas pelo aparelho durante $1 \mathrm{~h} 40 \mathrm{~m}$, na sala de aula que as professoras lecionavam. O espaço entre as duas medições foi de uma semana, sendo mantidas a mesma sala, mesmo horário e mesma disciplina lecionada, em ambos os momentos. Resultados: O parâmetro intensidade foi o único que apresentou diferença com o uso de amplificação de voz. Conclusão: $O$ uso da amplificação de voz durante a docência de professoras não disfônicas não interfere nos parâmetros acústicos de frequência fundamental, e nas medidas de dose vocal. A intensidade da voz é menor quando o professor faz uso de amplificação vocal.

\begin{abstract}
Objectives: Analyze the interference of using the voice amplifier in vocal dose of non-dysphonic teachers. Methods: This is an experimental study comparing people from the same ambience compound for 20 teachers from elementary school at municipal service in Belo Horizonte/MG. After participants' consent, they were requested to answer the vocal symptom scale questioner (ESV) and later participated from two different moments of the study which was automatically selected. In the first moment, the participants used only the vocal dosimeter and in the second, they used the vocal dosimeter and the voice amplifier. The measurements were recorded by the device for $1 \mathrm{~h} 40 \mathrm{~m}$, in the classroom that the teachers taught. The time between the two measurements was one week, with the same room, the same time and the same discipline being taught, at both times. Results: The intensity parameter was the only one that showed difference with the use of the voice amplifier. Conclusion: Use voice amplification while non dysphonic teachers are teaching doesn't affect the fundamental frequency and vocal dose measure in the acoustics parameters. The vocal intensity is smaller when teacher uses the vocal amplification.
\end{abstract}

Trabalho realizado no Programa de Pós-graduação em Ciências Fonoaudiológicas, Universidade Federal de Minas Gerais - UFMG - Belo Horizonte (MG), Brasil.

${ }^{1}$ Programa de Pós-graduação em Ciências Fonoaudiológicas, Departamento de Fonoaudiologia, Faculdade de Medicina, Universidade Federal de Minas Gerais - UFMG - Belo Horizonte (MG), Brasil.

${ }^{2}$ Hospital João XXIII - Belo Horizonte (MG), Brasil.

${ }^{3}$ Departamento de Fonoaudiologia, Faculdade de Medicina, Universidade Federal de Minas Gerais - UFMG Belo Horizonte, MG, Brasil.

Fontes de financiamento: Coordenação de Aperfeiçoamento de Pessoal de Nível Superior - Brasil (CAPES) Código de Financiamento 001. Conselho Nacional de Desenvolvimento Científico e Tecnológico-Brasil (CNPq) $\left(\mathrm{n}^{\circ} 309108 / 2019-5\right)$.

Conflito de interesses: nada a declarar. 


\section{INTRODUÇÃO}

O grau de exposição dos tecidos das PPVV à vibração pode ser expresso como o valor da dose vocal, que é calculada a partir de três parâmetros: amplitude, frequência e tempo ${ }^{(1)}$. Esses parâmetros são a base para o cálculo da dose vocal no monitoramento da fonação.

A literatura ${ }^{(1,2)}$ define cinco tipos de dose vocal: A dose de tempo (Dt) que quantifica o tempo total de vibração das PPVV durante a fala e é medida em segundos; a dose cíclica (Dc) que quantifica o número total de períodos de oscilação concluídos pelas PPVV ao longo do tempo e é medida em número de ciclos; a dose da distância (Dd) que quantifica a distância total percorrida pelo tecido das PPVV na trajetória cíclica durante a vibração e é medida em metros; a dose de dissipação de energia (De), que leva em conta o fator de agitação térmica do tecido no interior das PPVV e mede a quantidade de calor produzido durante a vibração das PPVV; e a dose de energia irradiada (Dr) ${ }^{(1,2)}$ que relaciona o consumo de energia nas PPVV à energia acústica irradiada na boca. Os estudos sobre dose vocal têm utilizado como principais medidas: a dose temporal, a dose cíclica e a dose de distância ${ }^{(3)}$.

Pesquisas mostram que a dose vocal aumenta em situações de fala com maior variação prosódica ${ }^{(1)}$; na docência ${ }^{(4)}$, especialmente em professores do ensino infantil ${ }^{(5)}$; nos ensaios da voz cantada e na docência do canto ${ }^{(6)}$; em situações de sobrecarga vocal como a fala em forte intensidade ${ }^{(7)}$; em ambientes com maior intensidade de ruído ${ }^{(8)}$; e nos indivíduos com quadros de disfonia ${ }^{(9)}$, principalmente as de base comportamental ${ }^{(10)}$. Em contrapartida, a dose vocal diminui em situações de repouso da voz ${ }^{(11)}$; em indivíduos com presbifonia $^{(12)}$ e com o uso de amplificação vocal durante a atividade de docência em professores do ensino fundamental ${ }^{(13)}$, em professores disfônicos ${ }^{(14)} \mathrm{e}$ em professores de canto ${ }^{(15)}$.

Revisão de literatura evidencia que existe uma alta prevalência de disfonia em professores, duas a três vezes mais frequente do que na população em geral ${ }^{(16,17)}$. No Brasil, pesquisas com docentes evidenciam uma prevalência de disfonia que varia de $11,6 \%$ a $89 \%{ }^{(18)}$. Alterações vocais não se limitam ao território brasileiro, na Europa, a prevalência de disfonia em professores é de 57\% na Espanha $^{(19)}$ e 51\% na Finlândia ${ }^{(20)}$, e de 11\% nos Estados Unidos ${ }^{(21)}$.

As disfonias são frequentemente associadas às inadequadas condições de trabalho do docente, e alguns dos fatores de risco elucidados são altos níveis de ruído externos e internos à sala de aula provocando o uso continuado da voz em forte intensidade ${ }^{(17,22)}$. Pesquisa ${ }^{(23)}$ mostra que a quantidade de energia sonora (Leq) encontrada em salas de aula vazias e mobiliadas varia de 54,51 a 74,04 $\mathrm{dB}(\mathrm{A})$, com mediana de $60 \mathrm{~dB}(\mathrm{~A})$, valores considerados elevados para a Norma brasileira para ruído em ambientes (NBR 10.152)(24).

$\mathrm{O}$ uso prolongado da voz no ambiente de trabalho associado a condições ambientais desfavoráveis, como o ruído ambiental elevado, pode expor os tecidos da laringe e das pregas vocais (PPVV) a vibrações excessivas, o que pode contribuir para o desenvolvimento de quadros de disfonia ${ }^{(9)}$, resultando em uma preocupação no âmbito da saúde pública na população docente. Para estes profissionais, a disfonia pode interferir na sobrevivência dos indivíduos no mercado de trabalho, representar a impossibilidade em exercer a profissão, acarretando faltas ao trabalho, diminuição de rendimento, e até mesmo na necessidade de mudança de profissão ${ }^{(22)}$.
O uso do amplificador vocal por professores é apresentado pela literatura como uma estratégia de proteção da voz ${ }^{(21-25)}$. Apesar da literatura evidenciar o impacto positivo do uso deste instrumento na qualidade vocal ${ }^{(26-28)}$, em parâmetros acústicos ${ }^{(26,28)}$, e na autopercepção da $v^{2} z^{(27,29)}$ dos docentes, pesquisas que analisaram a resposta do amplificador na dose vocal dos professores ainda são incipientes, e avaliaram professores do ensino fundamental ${ }^{(13)}$, professores disfônicos ${ }^{(14)}$ e professores de canto ${ }^{(15)}$.

O amplificador de voz permite ao professor falar em uma intensidade inferior quando comparada a não utilização do amplificador vocal(25), o que reduz a sobrecarga laríngea e o excesso de exposição dos tecidos das PPVV à vibração ${ }^{(26)}$, podendo minimizar a dose vocal deste grupo profissional.

Professores utilizam durante a docência uma intensidade vocal de 10 a 15 dBNPS acima do ruído ambiental ${ }^{(27)}$. O prejuízo no tecido das PPVV, causado pela força de colisão, é reduzido quando a intensidade da voz é diminuída pelo uso do amplificador vocal $^{(29)}$. A literatura mostra associação positiva entre o aumento da dose vocal em professores e o aumento do nível do ruído ambiental ${ }^{(8)}$. A literatura evidencia também que a presença de disfonia aumenta a dose vocal de professores ${ }^{(9)}$ e que os mesmos possuem o dobro do tempo de fonação quando comparados aos não profissionais da $\mathrm{voz}^{(30)}$.

Portanto, para o desenvolvimento de estratégias de prevenção e promoção da saúde da voz de professores, é importante investigar o impacto da amplificação vocal em docentes sem queixa de voz, o que pode contribuir para o enfrentamento do adoecimento vocal deste grupo profissional.

O objetivo desta pesquisa foi analisar se o uso do amplificador de voz interfere na dose vocal de professoras não disfônicas.

\section{MÉTODO}

Trata-se de um estudo experimental comparativo intrassujeitos, aprovado pelo Comitê de Ética em Pesquisa da Universidade Federal de Minas Gerais (UFMG) sob o número 47212615.1.0000.5149.

Para compor a amostra foram selecionadas 20 professoras do ensino fundamental, com idade de 27 a 45 anos (média=37,5; $\mathrm{DP}=6,2)$ de três escolas da Rede Municipal de Ensino de Belo Horizonte/MG (RME-BH), no período de julho a agosto de 2018. As escolas selecionadas apresentavam em média 16 salas de aulas, com turmas do ensino infantil, Fundamental I e II, e funcionavam nos períodos da manhã e da tarde.

Trata-se de uma amostra por conveniência e, para a determinação do número de professoras participantes da pesquisa, foi utilizado o programa estatístico G.Power $3.1^{\circledR}$. O tamanho da amostra foi baseado no estudo de Rabelo et al.(2019) ${ }^{(8)}$, com os resultados da comparação da dose cíclica de mulheres em situações acústicas diferenciadas, com e sem ruído. O cálculo amostral determinou o número de 15 professoras em cada grupo, considerando o teste Wilcoxon para amostra pareada com poder do estudo de $95 \%$, alfa igual a 0,05 e tamanho do efeito de 1.05 .

As participantes receberam uma visita das pesquisadoras nas escolas onde lecionam, e foram informadas sobre o objetivo da pesquisa e os critérios para participação no estudo. As professoras que livremente expressaram seu consentimento, aceitando participar voluntariamente da pesquisa, foram esclarecidas sobre os procedimentos de coleta de dados e a finalidade do uso das informações coletadas, por meio da assinatura do Termo de 
consentimento Livre e esclarecido (TCLE). Todas as professoras que apresentavam autopercepção positiva da qualidade vocal (referiram ter voz boa ou muito boa) e autorreferiram ausência de sintomas vocais (fadiga e/ou desconforto), foram convidadas a realizar avaliação fonoaudiológica e otorrinolaringológica para participarem da pesquisa. Todas as 20 professoras do ensino fundamental convidadas a participar da pesquisa foram incluídas no estudo e finalizaram a coleta.

A avaliação fonoaudiológica constou da análise perceptivo-auditiva do grau geral da disfonia em uma escala Likert de quatro pontos, nas tarefas de vogal /a/ sustentada de forma habitual e da fala encadeada (dias da semana). Os registros vocais foram coletados utilizando-se microfone unidirecional, condensador, da marca Shure $^{\circledR}$, modelo SM86, posicionado a $10 \mathrm{~cm}$ de distância da boca de cada participante, ligado diretamente em notebook (Toshiba Satellite 1800/1850), placa de som Sound blaster, por meio do programa Sonic Foundry Soundforge 6.0, mono, 16 bits, frequência de amostragem $44.100 \mathrm{~Hz}$, na própria escola, em ambiente silente. Considerou-se como qualidade vocal neutra o grau geral da disfonia graduado em zero. A avaliação fonoaudiológica foi realizada por uma das pesquisadoras, com cinco anos de experiência em análise perceptivo-auditiva. Para esta análise, a avaliadora reproduziu as vozes quantas vezes julgou necessário, utilizando fone de ouvido supra-auricular modelo Multilaser Vibe Headphone estéreo.

A avaliação otorrinolaringológica foi realizada por médicos otorrinolaringologistas, por meio de videolaringoscopia. Considerou-se como exame laríngeo normal aqueles que apresentaram ausência de lesões nas pregas vocais e coaptação glótica completa. A presença de fenda triangular posterior nas mulheres foi considerada fisiológica ${ }^{(31)}$. Foram analisados os exames de videolaringoscopia realizados nas avaliações periódicas dos professores da RME-BH, há pelo menos quatro meses da data da coleta da pesquisa.

Para análise dos sintomas vocais as professoras responderam um instrumento de autoavaliação de voz (ESV), que contempla informações de funcionalidade, impacto emocional e físico em decorrência da presença de sintomas vocais. O ESV é considerado um protocolo simples e de fácil aplicação e interpretação, composto por 30 questões e com ponte de corte igual ou superior a 16 pontos, para definir a presença de queixas vocais ${ }^{(32)}$. As participantes pontuaram de 0 a 15 indicando ausência de queixas vocais (média $=10,6 ; \mathrm{DP}=3,2$ ).

Foram incluídas no estudo professoras do sexo feminino; que lecionavam apenas dentro de sala de aula; com idade entre 18 e 45 anos, por este ser o período de maior estabilidade vocal; protocolo de Escala de sintomas vocais $(\mathrm{ESV})^{(32)}$ inferior a 16 pontos e autoavaliação de ausência de sintomas vocais; exame laríngeo normal verificado na avaliação otorrinolaringológica de videolaringoscopia; e qualidade vocal neutra observada na avaliação fonoaudiológica. Os critérios de exclusão adotados foram apresentar queixa autodeclarada de distúrbio auditivo ou pulmonar, fumantes, grávidas ou no período pré-menstrual ou menstrual.

$\mathrm{O}$ instrumento utilizado para medir a dose vocal das professoras foi um dosímetro vocal da marca $\operatorname{Vox}_{\log ^{\mathbb{R}}}$ da Sonvox modelo 3.1, composto por um microfone, uma unidade portátil que armazena os dados vocais e um acelerômetro (Figura 1), posicionado na região do pescoço, próximo à cartilagem tireóidea (Figura 2).

Para amplificação da voz foi utilizado o amplificador de voz portátil da marca $\mathrm{BOAS}^{\circledR}$, com alcance de até 200 metros, potência de saída de $5 \mathrm{w}$ e, frequência de resposta de $100 \mathrm{hz}$ a $13000 \mathrm{~Hz}$ (Figura 3).

As participantes foram monitoradas em dois dias distintos, definindo dois momentos de gravação. No momento 1 (M1) as professoras utilizaram o dosímetro vocal na sala de aula onde lecionavam, tendo a duração de dois horários de aula (1h40min). No momento 2 (M2) as participantes utilizaram o dosímetro vocal na mesma sala de aula, tendo a mesma duração de dois horários de aula (1h40min), fazendo uso concomitante do amplificador de voz da marca BOAS ${ }^{\circledR}$.

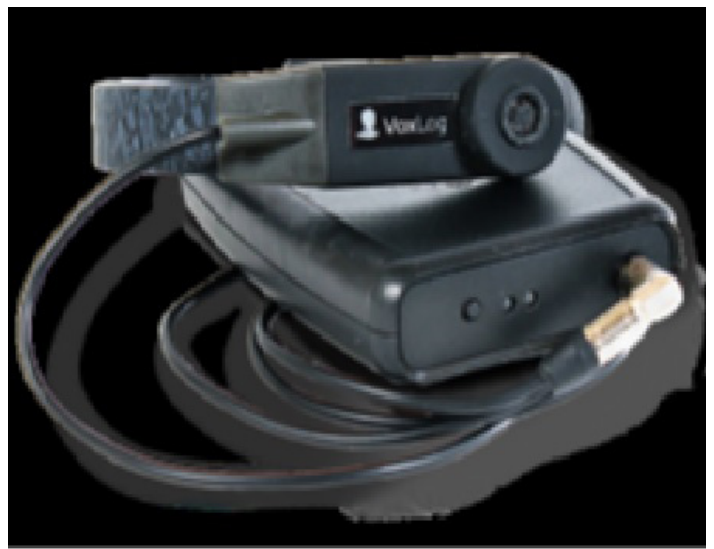

Fonte: http://www.sonvox.com/VoxLog_technical_document.pdf

Figura 1. dosímetro vocal da marca VoxLog ${ }^{\circledR}$ da Sonvox modelo 3.1

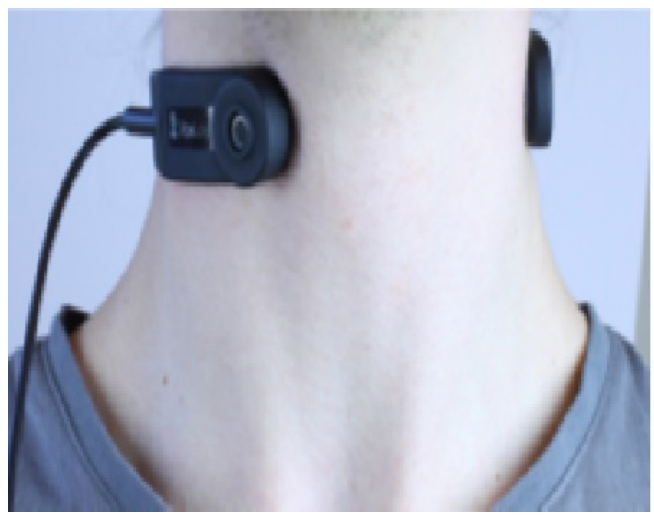

Fonte: http://www.sonvox.com/VoxLog_technical_document.pdf Figura 2. colocação do equipamento

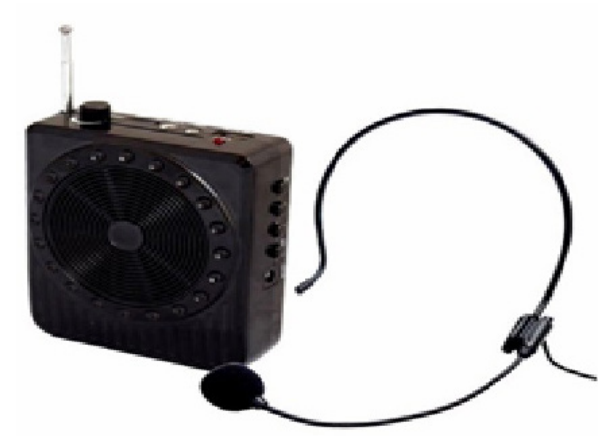

Fonte: https://www.xfort.com.br/2018/07/amplificadordevoz-ap50.html Figura 3. Amplificador de voz da marca BOAS ${ }^{\circledR}$ 
As professoras não receberam treinamento vocal específico para utilizar o amplificador vocal. Foram orientadas em como manipular o equipamento, como utilizá-lo durante as aulas, e qual o objetivo do uso do amplificador de voz. Cada professora testou e utilizou o equipamento com uma das pesquisadoras, e foi questionada sobre alguma dúvida relacionada à manipulação e uso do equipamento.

O ruído ambiental foi aferido pelo medidor de nível de pressão sonora com data-logger da marca Instrutherm ${ }^{\circledR}$ modelo DEC-490 com microfone tipo 2. As medições foram realizadas nas bandas de oitava que abrangem as frequências de $63 \mathrm{~Hz}$ a $8 \mathrm{KHz}$, com as salas de aula mobiliadas e com atividades escolares ocorrendo normalmente, inclusive nas salas de aula adjacentes. $\mathrm{O}$ medidor de nível de pressão sonora foi posicionado no centro da sala, a $1,2 \mathrm{~m}$ do chão, $0,5 \mathrm{~m}$ de objetos móveis e $1 \mathrm{~m}$ das paredes e objetos fixos, e a aproximadamente $1,0 \mathrm{~m}$ de distância do professor (ANSI S12.60, 2010).

A partir dos valores medidos na sala de aula, foi calculado o Nível Sonoro Contínuo Equivalente (Leq), que é definido como sendo o nível de som contínuo que tenha a mesma energia acústica do som flutuante que está sendo medido em um determinado local. O cálculo é feito integrando-se a variação da pressão no tempo. A média do ruído ambiental foi de 72,5 dBNPS $(\mathrm{DP}=5,2)$ no momento sem o amplificador de voz (M1), e de 72,8 dBNPS $(\mathrm{DP}=5,2)$ no momento com o amplificador de voz (M2), sem diferença de nível de ruído entre os dois momentos $(\mathrm{p}=0,06)$.

Os momentos M1 e M2 foram aleatorizados, e definidos para cada professora por meio de sorteio. $\mathrm{O}$ intervalo de distância entre os dois momentos do estudo foi de uma semana. Para homogeneizar a amostra, as gravações de M1 e M2 aconteceram no mesmo dia da semana, horário de aula, turma de alunos, e disciplina lecionada.

A coleta foi realizada na própria escola onde as participantes lecionam, sempre no turno da manhã, nas primeiras aulas do dia. A média do tempo da primeira gravação das vozes (M1) com o dosímetro vocal foi de 105,4 minutos (DP 4,5), e da segunda gravação (M2) foi de 106,3 minutos (DP 5,0), o que demonstra estabilidade de tempo de gravação nos dois momentos $(p=0,51)$.

Os dados vocais coletados foram analisados no computador por meio de software específico do equipamento $\operatorname{Vox}_{\log }{ }^{\mathbb{R}} \mathrm{e}$ constituem da análise dos seguintes parâmetros:

1. Intensidade vocal: representa a quantidade de energia do som produzido, medida em dBNPS ${ }^{(1)}$

2. Frequência fundamental $\left(\mathrm{f}_{0}\right)$ : é o número de ondas sonoras por unidade de tempo e é medida em $\mathrm{Hz}^{2}$.

3. Porcentagem de fonação: indica o tempo no qual a fonação é produzida, comparado com o tempo decorrido do período monitorado, medido em porcentagem ${ }^{(2,13)}$ :

$\frac{\text { Tempo de fonação X } 100}{\text { Tempo de gravação }}=\%$

4. Dose cíclica: quantifica o número de oscilações das pregas vocais durante o período gravado. É calculada a partir do tempo de fonação e da média da $\mathrm{f}_{0}$. $\mathrm{O}$ valor, em milhares de ciclos, é definido por ${ }^{(2)}$ :

$D c=\int_{0}^{t m} K v F 0 d t c i c l o s$ onde $t_{p}$ é o tempo de desempenho, ou seja, o tempo em que o indivíduo utiliza a sua voz, falando ou cantando, F0 é a frequência fundamental das pregas vocais em $\operatorname{Hertz}(\mathrm{Hz})$ e $k_{v}$ é a função definida por 1 (para emissões com vibração das PPVV) ou 0 (para momentos sem produção vocal) $)^{(2)}$ :

\section{$k_{v}=\left\{\begin{array}{l}1(\text { presença de vibração das } P P V V) \\ 0(\text { ausência de vibração das } P P V V)\end{array}\right.$}

5. Dose da distância: é a distância total percorrida pelo tecido das pregas vocais na trajetória cíclica durante a vibração e depende não somente do tempo total de fonação e frequência fundamental, mas também da amplitude da vibração da prega vocal e, portanto, da intensidade vocal (dB NPS). O valor desta dose, em metros, é definido por ${ }^{(2)}$ :

$D_{d}=4 \int_{0}^{t p} \mathrm{k}_{\mathrm{v}} A F O \mathrm{dt}$

em que F0 é a frequência fundamental das pregas vocais $(\mathrm{Hz})$ e A é a amplitude de vibração das pregas vocais no topo e base.

Para o cálculo da dose de distância pesquisadores desenvolveram um algoritmo, pois esta dose não é calculada pelo software do fabricante, como os outros parâmetros. $\mathrm{O}$ valor de $A$ pode ser determinado baseado nas regras empíricas derivadas ${ }^{(2)}$ :

$$
A=0,05 L_{o}\left[\left(P_{L}-P_{t h}\right) / P_{t h}\right]^{1 / 2} m,
$$

onde $L_{o}$ representa o comprimento das pregas vocais $(0,016 \mathrm{~m}$ para homens e $0,01 \mathrm{~m}$ para mulheres), $P_{L}$ é a pressão pulmonar e $P_{t h}$ é o limite de pressão fonatória. Para $P_{t h}$ é determinado ${ }^{(2)}$ :

$$
P_{t h}=0,14+0,06\left(F_{O} / F_{O N}\right)^{2} k P a,
$$

onde $\mathrm{F} 0$ é a frequência fundamental $\mathrm{e}_{O N}$ é a frequência fundamental nominal (120 Hz para homens e $190 \mathrm{~Hz}$ para mulheres). Para determinar a pressão pulmonar, esta foi derivada da medida do NPS a $50 \mathrm{~cm}$ da boca e é descrita como ${ }^{(2)}$ :

$$
P_{L}=P_{t h}+10^{(N P S-78,5) / 27,3} k P a
$$

A análise estatística dos dados foi realizada por meio do programa estatístico MINITAB versão 17. Primeiramente foi realizada uma análise descritiva dos dados com medidas de tendência central e dispersão. Posteriormente, foi utilizado o teste de Anderson-Darling para verificar a normalidade da amostra. Para comparação das medidas de intensidade com e sem o uso de amplificação de voz utilizou-se o teste não paramétrico de Wilcoxon pelo fato desta variável apresentar distribuição assimétrica. O teste T de Student pareado foi realizado para comparar as demais variáveis com distribuição normal nas situações com e sem o uso de amplificação de voz. Considerou-se o nível de confiança de 95\%.

\section{RESULTADOS}

O uso do amplificador de voz diminuiu os valores do parâmetro de intensidade vocal (Tabela 1). 
Tabela 1. Análise dos parâmetros vocais sem e com o uso do amplificador de voz em professoras sem disfonia

\begin{tabular}{|c|c|c|c|c|c|c|c|c|c|c|c|}
\hline \multirow{2}{*}{ Parâmetro } & \multicolumn{5}{|c|}{ Sem Amplificador de Voz } & \multicolumn{6}{|c|}{ Com Amplificador de Voz } \\
\hline & Média & $\mathrm{DP}$ & Mínimo & Mediana & Máximo & Média & $\mathrm{DP}$ & Mínimo & Mediana & Máximo & Valor $\mathrm{P}$ \\
\hline $\begin{array}{c}\text { Frequência } \\
\text { Fundamental (fo) }\end{array}$ & 281,9 & 32,9 & 216,7 & 280,7 & 368,6 & 270,7 & 30,8 & 210,14 & 265,9 & 353,4 & 0,188 \\
\hline Intensidade & 94,2 & 2,7 & 90,8 & 93,4 & 99,1 & 93,2 & 2,7 & 89,3 & 92,3 & 98,8 & $0,042^{*}$ \\
\hline $\begin{array}{l}\text { Porcentagem de } \\
\text { Fonação(\%) }\end{array}$ & 21,2 & 6,0 & 10,3 & 19,5 & 35,0 & 19,6 & 5,2 & 10,1 & 19,0 & 29,1 & 0,264 \\
\hline Dose cíclica & 306,9 & 107,8 & 118,0 & 320,0 & 488,0 & 284,9 & 97,7 & 103,0 & 273,5 & 457,0 & 0,417 \\
\hline Dose de distância & 1,8 & 0,7 & 0,7 & 1,8 & 3,6 & 1,8 & 0,8 & 0,5 & 1,8 & 3,3 & 0,810 \\
\hline
\end{tabular}

${ }^{*}$ P-Valor $<0,05$. Teste de Wilcoxon Teste T de Student pareado

\section{DISCUSSÃO}

Este estudo consistiu em analisar se o uso do amplificador de voz interfere na dose vocal de 20 professoras não disfônicas de escolas da Rede Municipal de Ensino. Os dados encontrados demonstram que a intensidade vocal das professoras não disfônicas, sofreu redução significativa com o uso do amplificador de voz. Os dados fonatórios de $\mathrm{f}_{0}$, porcentagem de fonação, dose cíclica, e dose de distância não apresentaram diferenças quando comparados os momentos sem amplificação e com amplificação.

Os professores têm sua voz como instrumento imprescindível para o desenvolvimento do ensino, e o comprometimento da capacidade vocal é um dos motivos mais comuns para o aumento do índice de absenteísmo relacionado à doença ${ }^{(33)}$. Portanto, é de grande importância para o fonoaudiólogo avaliar a função vocal em relação à real situação de ensino do professor. Neste estudo o foco foi na comparação da dose vocal com e sem o uso do amplificador de voz em uma situação real de ensino.

A literatura apresenta poucos estudos que analisam a associação da diminuição da dose vocal de professores com a amplificação de voz. Pode-se mencionar pesquisa em situações de aulas de canto $^{(15)}$, um estudo de caso com dois professores ${ }^{(13)}$ e por fim uma pesquisa com 15 professoras disfônicas ${ }^{(14)}$. Entretanto, não descreve estudos com professoras não disfônicas e amplificação de voz, e suas correlações com a dose vocal.

Uma pesquisa com professoras vocalmente saudáveis, em situação real de ensino, observou valores de $\mathrm{f}_{0}$ de 298,6 , e na realização de um teste de sobrecarga vocal (vocal loading) encontrou valores de $269,4 \mathrm{~Hz}^{(7)}$. Os resultados de $\mathrm{f}_{0}$ observados se aproximam dos achados do presente estudo.

As pesquisas evidenciam que a $\mathrm{f}_{0}$ se correlaciona com a intensidade na voz falada ${ }^{(8)}$. $\mathrm{Af}_{0}$ tende a se elevar com o aumento da intensidade, o que confirma os achados deste estudo sem o uso do amplificador de voz ${ }^{(27,34)}$. Pesquisas observaram que a $\mathrm{f}_{0}$ e a intensidade aumentam com a presença de ruído ambiental $^{(13,14)}$, o que pode estar relacionado ao aumento da atividade muscular, devido à carga vocal imposta durante a jornada de trabalho. Os professores queixam-se da necessidade de falar em forte intensidade para serem ouvidos/compreendidos em ambientes ruidosos e em condições acústicas inadequadas das salas de aula ${ }^{(17)}$.

Os resultados do presente estudo revelaram ainda que, a diminuição da intensidade da voz com o uso do amplificador, não foi suficiente para diminuir estatisticamente a $\mathrm{f}_{0}$ das participantes.
A diminuição da intensidade observada na pesquisa foi decorrente do uso do amplificador de voz, o que já era esperado, pois, o equipamento tem a função de modificar a intensidade vocal uma vez que o indivíduo utiliza a fala de forma mais confortável ${ }^{(25)}$. Além disso, o amplificador vocal é um dispositivo que pode contribuir para o conforto fonatório e longevidade vocal do profissional da voz, proporcionando diminuição de sintomas de desconforto vocal, e consequentemente melhorando o quadro clínico do professor ${ }^{(29)}$.

O uso do amplificador de voz promoveu uma leve redução da intensidade da voz das professoras de 94,2 dBNPS para 93,2 dBNPS. Limites seguros de intensidade da voz, em situações de uso profissional e/ou social, ainda não foram estabelecidos pela literatura, considerando os aspectos idiossincráticos da disfonia. A literatura ${ }^{(35)}$ define que em uma mulher com valor médio de $\mathrm{f}_{0}$ de $300 \mathrm{~Hz}$, um aumento de $6 \mathrm{~dB}$ na intensidade habitual da voz, vai modificar seu esforço vocal autorreferido de nada de esforço a uma sensação de esforço extremo, sugerindo que pequenas elevações da intensidade da voz apresentam impacto negativo na autopercepção vocal.

Estudos futuros são necessários para compreender se o pequeno aumento da intensidade, observado nesta pesquisa, é clinicamente relevante para impactar na saúde vocal de professoras sem queixa de voz.

A porcentagem de fonação está relacionada ao tempo de vibração das pregas vocais ao longo do período de gravação(1). Pesquisas demostram que seus valores não são dependentes apenas do tempo em que a voz foi gravada, mas fatores como a presença de disfonia em professores ${ }^{(9)}$, e em mulheres com disfonia comportamental ${ }^{(10)}$, aumentam a porcentagem de fonação. Os achados desta pesquisa demonstraram que o uso do amplificador de voz não diminuiu o tempo de fonação das professoras. Tais resultados corroboram com os achados de duas pesquisas que observaram que a amplificação vocal não altera a porcentagem de fonação de docentes durante as atividades letivas ${ }^{(13,14)}$. Portanto, a amplificação vocal não parece ser um fator importante para alterar a quantidade de fala do professor durante a atividade docente.

Em contrapartida, um estudo com professores de música demonstrou que o uso do amplificador de voz diminuiu a porcentagem de fonação ${ }^{(15)}$. Tais resultados sugerem que o uso do amplificador vocal na docência da voz cantada tende a funcionar de forma distinta em termos de tempo de fonação. Nos professores de música esta redução provavelmente está 
associada ao retorno auditivo positivo, possibilitando um melhor controle da voz cantada ${ }^{(15)}$.

Assim como a porcentagem de fonação não sofreu influência do amplificador de voz, a dose cíclica e a dose de distância também não modificaram. Como estes parâmetros são dependentes dos valores de $\mathrm{f}_{0}$ e da porcentagem de fonação, tais resultados já eram esperados, e sugerem que a amplificação vocal não interfere na quantidade de vibrações das pregas vocais de professoras não disfônicas.

A literatura evidencia que o uso do amplificador de voz em professoras disfônicas diminui a dose cíclica e a dose de distância, o que indica uma redução na força de colisão e nos riscos de danos às pregas vocais destes docentes ${ }^{(14)}$. Tais dados sugerem que esse recurso funciona de forma distinta para a população de professores disfônicos.

Uma pesquisa com cinco professoras com disfonia e cinco professoras sem alteração de voz durante a atividade letiva demonstrou que professoras disfônicas apresentam maior tempo de fonação e de dose cíclica quando comparadas com professoras sem alteração vocal ${ }^{(9)}$, sugerindo que professoras sem alterações de voz apresentam uma menor demanda vocal durante a atividade letiva. É lícito supor que o amplificador vocal tenha uma influência mais específica na intensidade da voz em professores sem alteração vocal, e não acarrete modificações importantes nos parâmetros de dose vocal.

Tal suposição é reforçada por um estudo de caso que avaliou um professor disfônico e outro sem alteração de $v_{0 z} z^{(13)}$ e observou que o professor disfônico se beneficiou mais do amplificador de voz do que o professor não disfônico. Tais resultados sugerem um efeito terapêutico do uso do amplificador vocal para o grupo dos professores disfônicos, enquanto para o grupo dos não disfônicos, o amplificador vocal parece ser um instrumento de prevenção das alterações vocais diante da diminuição da intensidade vocal utilizada durante a docência.

O professor que trabalha com limitação na realização das suas atividades devido à voz, não desempenha satisfatoriamente sua função de dar aulas. O significado para o professor diante desse cenário pode ser a desmotivação, absenteísmos ou abandono da profissão ${ }^{(36)}$. O principal motivo no Brasil de absenteísmo na categoria é o distúrbio vocal, seguidos dos problemas respiratórios e emocionais ${ }^{(33)}$.

A elaboração de ações de vigilância dos ambientes e processos de trabalho deve visar a intervenção precoce para evitar o agravamento do problema de voz e consequentes faltas recorrentes no trabalho docente ${ }^{(32)}$. Nesse sentido, o presente trabalho sugere que o amplificador de voz, por reduzir a intensidade vocal do professor, pode ser uma ferramenta disponível para a prevenção de disfonias em docentes. Pesquisa ${ }^{(35)}$ observou que, na elaboração de um modelo multivariado para determinar um índice de fadiga vocal, o aumento da intensidade da voz explicou $66 \%$ do modelo. Portanto, o aumento da intensidade da voz tem uma alta correlação com a presença de fadiga vocal.

Lançar mão de uma única ferramenta para atingir trabalhadores com diferentes demandas é de grande valia para a saúde pública. O amplificador vocal quando utilizado por grupos distintos (sem disfonia e com disfonia) apresentou repercussões específicas para cada grupo. Para docentes não disfônicos, avaliados nesta pesquisa, a intensidade vocal apresentou diminuição, resultado que sugere a possibilidade de manutenção da qualidade da voz ao longo da docência. Enquanto a literatura ${ }^{(14)}$ demonstra que o grupo de professores disfônicos apresenta redução dos parâmetros de dose vocal, o que pode indicar efeito terapêutico para a alteração de voz.

No Brasil, políticas públicas foram estabelecidas para o enfrentamento da poluição sonora no país (NBR 10.152)(24). No entanto, faz-se necessário ampliar o conhecimento sobre a relação entre o ruído nas escolas, as características sociodemográficas e as condições de trabalho de professores, a fim de auxiliar nas propostas de intervenção.

Os níveis de ruído mensurados nas salas de aula das participantes desta pesquisa foram em torno de $72 \mathrm{dBNPS}$, valores próximos aos indicados pela literatura ${ }^{(23)}$, e considerados elevados para a Norma brasileira para ruído em ambientes (NBR 10.152)(24). Avaliar a acústica das salas de aula para se estabelecer adequadas indicações de equipamentos de amplificação de voz ${ }^{(37)}$, além de orientações sobre a importância da redução do ruído no ambiente escolar objetivando a saúde vocal dos professores, e o processo de aprendizado dos discentes, são aspectos importantes para a saúde escolar. Ações positivas e políticas públicas que visem a saúde vocal dos professores devem enfatizar os aspectos ergonômicos e organizacionais do trabalho desta classe profissional.

É preciso ir além das capacitações de caráter individual e comportamental, e criar espaços de discussões para buscar soluções a fim de diminuir a sobrecarga vocal atrelada às exigências e desafios encontrados pelos professores, principalmente relacionados às condições do ambiente de trabalho.

Como limitações desta pesquisa enumeramos os aspectos relacionados ao tempo de gravação; ausência de um Programa estruturado de treinamento para utilização dos amplificadores de voz; o tipo de delineamento da pesquisa com comparação intrassujeitos; e a utilização da análise perceptivo-auditiva da qualidade vocal realizada apenas por uma das pesquisadoras. As professoras foram avaliadas durante duas aulas consecutivas (1h40min), em dois momentos distintos (com e sem amplificação vocal). Pesquisas com coletas em períodos mais prolongados, e em mais dias da semana são importantes, principalmente pelo fato dos valores dos parâmetros de frequência fundamental, porcentagem de fonação e dose cíclica apresentarem redução do valor médio quando comparados os momentos sem amplificação e com amplificação, porém sem significância estatística.

Pesquisas futuras que analisem o efeito de um Programa de treinamento de utilização do amplificador de voz em professores são importantes para avaliar o impacto destas orientações nos resultados destes equipamentos na saúde da voz dos docentes.

Estudos futuros, com maior força de evidência científica, do tipo ensaios clínicos controlados e randomizados, são importantes para melhor compreensão da amplificação sonora em professores não disfônicos, para elaboração de protocolos de promoção e prevenção da saúde vocal

\section{CONCLUSÃO}

O uso do amplificador de voz durante a docência em professoras não disfônicas diminui a intensidade da voz e não 
interfere nos parâmetros acústicos de frequência fundamental e nas medidas de dose vocal.

Os resultados desta pesquisa sugerem que professores não disfônicos podem ser beneficiados com o uso de amplificação sonora, pela diminuição da intensidade vocal, o que provavelmente pode ser um fator protetor da saúde vocal deste grupo profissional.

\section{REFERÊNCIAS}

1. Titze IR, Svec JG, Popolo PS. Vocal dose measures: quantifying accumulated vibration exposure in vocal fold tissues. J Speech Lang Hear Res. 2003;46(4):919-32. http://dx.doi.org/10.1044/1092-4388(2003/072). PMid: 12959470.

2. Švec JG, Popolo PS, Titze IR. Measurement of vocal doses in speech: experimental procedure and signal processing. Logoped Phoniatr Vocol. 2003;28(4):181-92. http://dx.doi.org/10.1080/14015430310018892. PMid:14686546.

3. Assad JP, Magalhães MC, Santos JN, Gama ACC. Vocal dose: an integrative literature review. Rev CEFAC. 2017;19(3):429-38. http://dx.doi. org/10.1590/1982-021620171932617.

4. Titze IR, Hunter EJ, Svec JG. Voicing and silence periods in daily and weekly vocalizations of teachers. J Acoust Soc Am. 2007;121(1):469-78. http://dx.doi.org/10.1121/1.2390676. PMid:17297801.

5. Remacle A, Morsomme D, Finck C. Comparison of vocal loading parameters in kindergarten and elementary school teachers. J Speech Lang Hear Res. 2014;57(2):406-15. http://dx.doi.org/10.1044/2013 JSLHR-S-12-0351. PMid:24129011.

6. Schloneger MJ. Graduate student voice use and vocal efficiency in an opera rehearsal week: a case study. J Voice. 2011;25(6):265-73. http:// dx.doi.org/10.1016/j.jvoice.2010.09.010. PMid:21429708.

7. Echternach M, Nusseck M, Dippold S, Spahn C, Richter B. Fundamental frequency, sound pressure level and vocal dose of a vocal loading test in comparison to a real teaching situation. Eur Arch Otorhinolaryngol. 2014;271(12):3263-8. http://dx.doi.org/10.1007/s00405-014-3200-6. PMid:25012705.

8. Rabelo ATV, Santos JN, Souza BO, Gama ACC, Castro Magalhães M. The Influence of Noise on the Vocal Dose in Women. J Voice. 2019;33(2):214-9. http://dx.doi.org/10.1016/j.jvoice.2017.10.025. PMid:29290547.

9. Gama ACC, Santos JN, Pedra EFP, Rabelo AT, Magalhães MC, Casas EB. Vocal dose in teachers: correlation with dysphonia. CoDAS. 2016;28(2):1902. http://dx.doi.org/10.1590/2317-1782/20162015156. PMid:27191884.

10. Mehta DD, Van Stan JH, Zañartu M, Ghassemi M, Guttag JV, Espinoza $\mathrm{VM}$, et al. Using Ambulatory Voice Monitoring to Investigate Common Voice Disorders: research Update. Front Bioeng Biotechnol. 2015;3(155):114. http://dx.doi.org/10.3389/fbioe.2015.00155. PMid:26528472.

11. Misono S, Banks K, Gaillard P, Goding GS Jr, Yueh B. The clinical utility of vocal dosimetry for assessing voice rest. Laryngoscope. 2015;125(1):1716. http://dx.doi.org/10.1002/lary.24887. PMid:25137621.

12. Ziegler A, Hapner ER. Vocal dose in older adults with Presbyphonia: An analytic, cross-sectional study. J Voice. 2018;97(18):30320-5. PMid:30322822.

13. Gaskill CS, O’Brien SG, Tinter SR. The effect of voice amplification on occupational vocal dose in elementary school teachers. J Voice. 2012;26(5):667.e19-27. http://dx.doi.org/10.1016/j.jvoice.2011.10.010. PMid:22521533.

14. Assad JP, Gama ACC, Santos JN, Castro Magalhães M. The effects of amplification on vocal dose in teachers with Dysphonia. J Voice. 2019;33(1):739. http://dx.doi.org/10.1016/j.jvoice.2017.09.011. PMid:29122417.

15. Morrow SL, Connor NP. Voice amplification as a means of reducing vocal load for elementary music teachers. J Voice. 2011;25(4):441-6. http://dx.doi. org/10.1016/j.jvoice.2010.04.003. PMid:20655172.

16. Martins RHG, Pereira ERBN, Hidalgo CB, Tavares ELM. Voice Disorders in Teachers. A Review. J Voice. 2014;28(6):716-24. http://dx.doi.org/10.1016/j. jvoice.2014.02.008. PMid:24929935.
17. Limoeiro FMH, Ferreira AEM, Zambon F, Behlau M. Comparação da ocorrência de sinais e sintomas de alteração vocal e de desconforto no trato vocal em professores de diferentes níveis de ensino. CoDAS. 2019;31(2):e20180115. http://dx.doi.org/10.1590/2317-1782/20182018115. PMid:30892420.

18. Behlau M, Zambon F, Guerrieri AC, Roy N. Epidemiology of voice disorders in teachers and nonteachers in Brazil: prevalence and adverse effects. J Voice. 2012;26(5):665.e9-18. http://dx.doi.org/10.1016/j.jvoice.2011.09.010. PMid:22516316.

19. Preciado-López J, Perez-Fernandez C, Calzada-Uriondo M, Preciado-Ruiz P. Epidemiological study of voice disorders among teaching professionals of La Rioja, Spain. J Voice. 2008;22(4):489-508. http://dx.doi.org/10.1016/j. jvoice.2006.11.008. PMid:17574808.

20. Ilomäki I, Leppanen K, Kleemola L, Tyrmi J, Laukkanen AM, Vilkman E. Relationships between self-evaluations of voice and working conditions, background factors, and phoniatric findings in female teachers. Logoped Phoniatr Vocol. 2009;34(1):20-31. http://dx.doi.org/10.1080/14015430802042013. PMid: 19283550 .

21. Roy N, Merrill RM, Thibeault S, Parsa RA, Gray SD, Smith EM. Voice disorders in teachers and the general population. J Speech Lang Hear Res. 2004;47(2):281-93. http://dx.doi.org/10.1044/1092-4388(2004/023). PMid: 15157130 .

22. Jardim R, Barreto SM, Assunção AA. Condições de trabalho, qualidade de vida e disfonia entre docentes. Cad Saude Publica. 2007;33(10):2439-61. http://dx.doi.org/10.1590/S0102-311X2007001000019. PMid:17891304.

23. Rabelo ATV, Santos JN, Oliveira RC, Magalhães MC. Efeito das características acústicas de salas de aula na inteligibilidade de fala dos estudantes. CoDAS. 2014;26(5):360-6. http://dx.doi.org/10.1590/23171782/20142014026. PMid:25388068.

24. Associação Brasileira de Normas Técnicas. NBR 10.152: Níveis de ruído para conforto acústico. Rio de Janeiro: ABNT; 2017.

25. McCormick CA, Roy N. The ChatterVox portable voice amplifier: a means to vibration dose reduction? J Voice. 2002;16(4):502-8. http://dx.doi. org/10.1016/S0892-1997(02)00126-1. PMid:12512638.

26. Jónsdottir V, Laukkanen AM, Siikki I. Changes in teachers' voice quality during a working day with and without electric sound amplification. Folia Phoniatr Logop. 2003;55(5):267-80. http://dx.doi.org/10.1159/000072157. PMid: 12931060.

27. Bovo R, Trevisi P, Emanuelli E, Martini A. Voice amplification for primary school teachers with voice disorders: a randomized clinical trial. Int J Occup Med Environ Health. 2013;26(3):363-72. http://dx.doi.org/10.2478/ s13382-013-0115-1. PMid:23817868.

28. Masson MLV, Araújo TM. Protective Strategies Against Dysphonia in Teachers: Preliminary Results Comparing Voice Amplification and 0.9\% NaCl Nebulization. J Voice. 2018;32(2):257.e1-10. http://dx.doi. org/10.1016/j.jvoice.2017.04.013. PMid:28579158.

29. Teixeira LC, Behlau M. Comparison between vocal function exercises and voice amplification. J Voice. 2015;29(6):718-26. http://dx.doi.org/10.1016/j. jvoice.2014.12.012. PMid:26296853.

30. Hunter EJ, Titze IR. Variations in intensity, fundamental frequency, and voicing for teachers in occupational versus non-occupational settings. J Speech Lang Hear Res. 2010;53(4):862-75. http://dx.doi.org/10.1044/10924388(2009/09-0040). PMid:20689046.

31. Dedivitis RA, Tsuji DH. Manual Prático de Laringologia. $1^{\text {a }}$ ed. Rio de Janeiro: DI LIVROS; 2011.

32. Moreti F, Zambon F, Oliveira G, Behlau M. Cross-cultural adaptation, validation, and cutoff values of the Brazilian version of the Voice Symptom Scale. VoiSS. J Voice. 2014;28(4):458-68. http://dx.doi.org/10.1016/j. jvoice.2013.11.009. PMid:24560004.

33. Medeiros AM, Vieira MT. Ausência ao trabalho por distúrbio vocal de professores da Educação Básica no Brasil. Cad Saude Publica. 2019;35(1, Suppl. 1):e00171717. http://dx.doi.org/10.1590/0102-311x00171717. PMid:30994819.

34. Rantala LM, Hakala S, Holmqvist S, Sala E. Holmgvist, Sala E. Classroom Noise and Teachers' Voice Production. J Speech Lang Hear Res. 2015;58(5):1397-406. http://dx.doi.org/10.1044/2015_JSLHR-S-14-0248. PMid:26089145. 
35. Bottalico P. Speech Adjustments for Room Acoustics and Their Effects on Vocal Effort. J Voice. 2017;31(3):392.e1-12. http://dx.doi.org/10.1016/j. jvoice.2016.10.001. PMid:28029555.

36. Ferreira LL. Lições de professores sobre suas alegrias e dores no trabalho. Cad Saude Publica. 2019;35(1, Suppl 1):e00049018. http://dx.doi. org/10.1590/0102-311x00049018. PMid:30994815.

37. Cutiva LCC, Puglisi GE, Astolfi A, Carullo A. Four-day Follow-up Study on the Self-reported voice condition and noise condition of teachers: relationship between vocal parameters and classroom acoustics. J Voice. 2017;31(1):120. e1-8. http://dx.doi.org/10.1016/j.jvoice.2016.02.017. PMid:27427163.

\section{Contribuição dos autores}

EVSS coletou e analisou os dados e organizou o texto; IBB e ACCG revisaram criticamente o conteúdo do manuscrito. 